\title{
Alineamiento constructivo de las asignaturas de Comunicación y Métodos y Técnicas de Estudio
}

\section{Constructive alignment of Communication and Methods and Study Techniques subjects}

\section{RESUMEN}

Objetivos: Aplicar el alineamiento constructivo a las asignaturas de Comunicación, y Métodos y Técnicas de Estudio. Métodos: Investigación basada en un enfoque cuantitativo - cualitativo, con un alcance descriptivo; en la recolección y análisis de datos se aplicó un cuestionario estructurado sobre enfoques de aprendizaje, y plantillas de análisis de contenido. La muestra probabilística fue de 188 estudiantes de seis escuelas académicas de la Facultad de Ciencias de la Empresa de la Universidad Continental. Resultados: El 82,98\% de estudiantes de la muestra, presenta un enfoque profundo de aprendizaje. Este enfoque se desagrega en: intensidad baja $56,91 \%$, intensidad media $21,28 \%$ e intensidad alta $4,79 \%$; en tanto, el $17,02 \%$ de estudiantes, presenta un enfoque superficial. Producto del alineamiento constructivo de ambas asignaturas se construyen sus sílabos desde la percepción de la psicología cognitiva, estableciendo una coherencia interna entre sus competencias, contenidos, metodología y evaluación. Conclusiones: Los estudiantes de la Facultad de Ciencias de la Empresa tienen un enfoque profundo de aprendizaje, pero con una intensidad baja. La asignatura de Comunicación asume el enfoque de la psicología cognitiva, fusiona los aportes funcionales, procesuales y de contenido de las teorías lingüístico comunicativas, enfatizando el aprendizaje de estrategias y habilidades: hablar, escuchar, leer y escribir. La asignatura de Métodos y técnicas de estudio - o Habilidades y estrategias de aprendizaje - asume el enfoque de la psicología cognitiva; ejercita los procesos de observación, comparación, relación, clasificación, ordenamien-

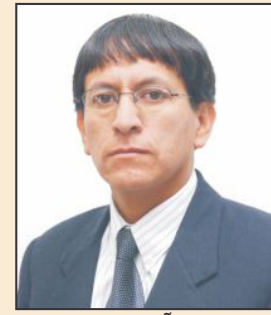

Fernando Ñaupari

to, análisis, síntesis y evaluación en el contexto de los estudios universitarios, ejercitándolas como herramientas cognitivas para generar aprendizajes.

Palabras clave: Alineamiento constructivo, comunicación, métodos de estudio, procesos cognitivos, estrategia.

1 Magíster en Educación. Investigador de la Universidad Continental. 


\section{ABSTRACT}

Objectives: To apply the constructive alignment to Communication and Methods and Study Techniques subjects. Methods: Research based on a quantitative qualitative approach, with a descriptive study, in the collection and data analysis we applied a structured questionnaire about learning approaches, and content analysis template. The probability sample was of 188 students from six academic schools of the Company Sciences faculty of the Continental University. Results: $82,98 \%$ of students in the sample, presents a deep approach of learning. This approach is broken down into: low intensity 56,91\%, medium intensity $21,28 \%$ and high intensity $4,79 \%$. Meanwhile, $17,02 \%$ of the students present a superficial approach. As a result of the constructive alignment of both subjects are build their syllabi from the perception of the cognitive psychology, establishing an internal coherence between their competencies, content, methodology and evaluation. Conclusions: Students of the Companies Sciences have a deep learning approach, but with a low intensity. The subject of communication takes the approach of cognitive psychology, merges functional, procedural and content inputs of communicative linguistic theories, emphasizing the learning of the strategies and skills: speaking, listening, reading and writing. The subject of Methods and Study techniques - or skills and learning strategies - takes the cognitive psychological approach; exercises the observation, comparison, relationship, classification, ordering, analysis, synthesis and evaluation processes in the context of university studies, exercising them as cognitive tools for generating learning.

Keywords: Constructive alignment, Communication, Study methods, cognitive processes, strategy.

\section{INTRODUCCIÓN}

En la literatura encontramos a Carrascal (1) quien relaciona enfoques de aprendizaje, contextos de enseñanza e implementación de tareas SOLO (Structure of the Observed Learning Outcome) para el desarrollo de competencias básicas en estudiantes de primer semestre de la Facultad de Ciencias Básicas e Ingenierías de la Universidad de Córdoba, Colombia. Con un diseño cuasi experimental con grupo control pre-test y post-test, observa los resultados de aprendizaje con y sin intervención del modelo instruccional de alineamiento constructivo e interactivo.

García (2), estudia los enfoques de aprendizaje de estudiantes de Magisterio y Psicopedagogía en la Universidad de Almería de España. Obtiene un porcentaje pequeño y similar de alumnado que adopta un enfoque predominantemente profundo o predominantemente superficial. La mayoría del estudiantado se muestra más motivado intrínsecamente, sin embargo utiliza estrategias memorísticas como comprensivas.

Manrique (3) estudia la evaluación procesual del currículo y la toma de decisiones para modificar el plan de estudios de una carrera. La investigación es de nivel descriptivo, utilizó un método mixto secuencial: cuantitativo seguido del cualitativo. Utilizó entrevistas y análisis de documentos. En el estudio cuantitativo, se aplicó el coeficiente de Pearson y el de Cohen; en el cualitativo, se utilizó matrices de análisis para establecer relaciones e inferencias. Concluye que los cambios en los planes de estudio obedecen a factores externos e internos, que impulsan o restringen la dinámica curricular procesual, como el informe evaluativo que se presenta al Consejo de Facultad.

Goded (4), plantea que un syllabus comunicativo construido en torno a la adquisición de conocimientos gramaticales tiene menos efectividad desde el punto de vista del aumento de la competencia comunicativa que un syllabus elaborado sobre la base de las necesidades comunicativas de los alum- 
nos, explicitada mediante su elección de temas e intereses de aprendizaje.

Maquilón (5), diseña y evalúa un Programa de Intervención para la mejora de las Habilidades de Aprendizaje de los estudiantes de la Universidad de Murcia. Enfatiza la necesidad que los estudiantes aborden sus aprendizajes desde una perspectiva profunda, lo que hace necesario el desarrollo de un paradigma de aprendizaje reflexivo, crítico e integral. Aplica un Cuestionario sobre Procesos de Estudio a partir del marco teórico de Biggs, para detectar necesidades y enfoques de aprendizaje. Se tiene que casi la mitad de ingresantes emplean predominantemente el enfoque superficial en sus tareas de aprendizaje.

El trabajo se justifica pues sus resultados permiten mejorar los procesos académicos de la Universidad Continental en lo relacionado a la actualización curricular, con la aplicación del alineamiento constructivo y el desarrollo de modelos cognitivos en la enseñanza-aprendizaje de los estudiantes.

La propuesta incluye la elaboración de sílabos que podrán ser utilizados por los docentes en la asignatura de Comunicación y, Métodos y Técnicas de estudio, con el desarrollo de habilidades comunicativas y habilidades de estudio, consideradas básicas y necesarias para el desempeño exitoso en las demás asignaturas.

Se busca que la universidad reconozca e identifique las condiciones con las que el joven ingresa, y de inmediato mejore sus estrategias de abordaje, inclusión y permanencia desde el ámbito académico. Resulta fundamental "alinear" las características y necesidades de aprendizaje del estudiante, sus procesos cognitivos, la intención del docente, su metodología y la evaluación de los aprendizajes esperados. Por ello se espera responder íqué características reúnen las asignaturas de Comunicación y, Métodos y técnicas de estudio con la aplicación del Alineamiento Constructivo?

El objetivo general de la investigación es: aplicar el Alineamiento Constructivo en las asignaturas de Comunicación y, Métodos y técnicas de estudio de la Universidad Continental de Huancayo. Los objetivos específicos son: describir el nivel de desarrollo de los procesos cognitivos de los estudiantes de la Universidad Continental de Huancayo; y alinear constructivamente las necesidades de aprendizaje, los propósitos, contenidos, estrategias y evaluación de las asignaturas de Comunicación y Métodos y técnicas de estudio.

\section{MATERIAL Y MÉTODOS}

La investigación se realizó desde un enfoque mixto secuencial. Por el lado cuantitativo, utilizó el método descriptivo; en el lado cualitativo, utilizó el análisis de contenido.

\section{Población y Muestra}

Para trabajar el primer objetivo específico, se tuvo una muestra estudiantil conformada por 188 estudiantes entre varones y mujeres, cuyo promedio de edad fue de 20 años, de las escuelas académicas profesionales de Administración y Marketing, Ciencias y Tecnologías de la Comunicación, Contabilidad, Derecho, Economía y Psicología; seleccionados con la técnica de muestreo probabilístico (estratificado). En todos los casos son estudiantes matriculados necesariamente, en las asignaturas de Comunicación y Métodos y Técnicas de Estudio, para el período agosto-diciembre 2011 -II.

Para trabajar el segundo objetivo específico. Del total de asignaturas que conforman el plan de estudios de las escuelas académicas profesionales de Administración y Marketing; Ciencias y Tecnologías de la Comunicación; Contabilidad, Derecho, Economía y Psicología; fueron seleccionadas -intencionalmente dos asignaturas de formación general o integral: Comunicación, y Métodos y Técnicas de Estudio, con las cuales se trabajó.

\section{Técnicas e instrumentos}

Para obtener información vinculada al 
desarrollo de los procesos cognitivos de los estudiantes se aplicó una encuesta a través del cuestionario sobre enfoques de aprendizaje (6). Adaptación, validación y evaluación de la invarianza factorial del cuestionario revisado de procesos de estudio en distintos contextos culturales: diseño del estudio y primeros resultados (The revised two-factor Study Process Questionnaire).

Para aplicar el alineamiento constructivo, a los propósitos, contenidos, estrategias y evaluación de las asignaturas de Comunicación y, Métodos y técnicas de estudio, se utilizó plantillas de análisis (7) a través del análisis de contenido (documentos curriculares) y la observación estructurada (sílabos).

\section{RESULTADOS}

La mayoría de los estudiantes de la muestra de estudio, como se presenta en la tabla 1, presentaban un enfoque profundo de aprendizaje (82,98\%). Ellos están interesados en la tarea académica y disfrutan llevándola a cabo; buscan el significado inherente y personalizan la tarea, haciéndola significativa para su propia experiencia y su mundo real; integran las partes o aspectos en un todo; tratan de teorizar y formar hipótesis.

Sólo $17,02 \%$ de estudiantes, presentaban un enfoque superficial de aprendizaje. Ellos, ven la tarea como una demanda que debe cumplirse, una imposición necesaria para cumplir algún objetivo; ven los aspectos o partes como discretas y no relacionadas unas con las otras o con otras tareas; se preocupan por el tiempo que toma hacer la tarea; evitan significados personales $\mathrm{o}$ de otro tipo que la tarea pueda tener; y se basan en la memorización, intentando reproducir aspectos superficiales.

De acuerdo con los resultados, tenemos en un extremo, un alto porcentaje de estudiantes con un enfoque profundo en sus aprendizajes, pero con intensidad baja $(56,91 \%)$. Al otro extremo, se observan estudiantes con un enfoque superficial en sus aprendizajes e intensidad baja $(17,02 \%)$.

En el tema de alineamiento constructivo de la asignatura de Comunicación, ésta se basó en la psicología cognitiva, fusionando además los aportes del enfoque funcional, procesual y, de contenido de la lingüística y la teoría de la comunicación. Debe enseñarse de modo integrado habilidades, estrategias y actitudes propias de un comunicador competente, a partir del interés o la obligación de realizar una carrera universitaria. Es fundamental aprender a usarla lengua: hablar, escuchar, leer y escribir; como una herramienta que permite comunicarse de modo efectivo y no como un conocimiento que se tengan que memorizar.

Se obtiene como producto el sílabo de

Tabla № 1: Frecuencia de enfoques de aprendizaje según su intensidad ingresantes - 2011-II

\begin{tabular}{rlrr}
\hline Enfoque & Intensidad & $\mathrm{fi}$ & $\%$ \\
\hline \multirow{3}{*}{ Profundo } & Baja & 107 & $56,91 \%$ \\
& Media & 40 & $21,28 \%$ \\
& Alta & 9 & $4,79 \%$ \\
Superficial & Baja & 32 & $17,02 \%$ \\
& Media & 0 & $0,00 \%$ \\
& Alta & 0 & $0,00 \%$ \\
Total & & 188 & $100,00 \%$ \\
\hline
\end{tabular}


Comunicación cuya sumilla es "Asignatura de naturaleza teórico - práctica y de formación general. Desarrolla en el estudiante habilidades, estrategias y actitudes relacionadas a la expresión y comprensión de textos académicos orales y escritos necesarios para un mejor desempeño personal y académico. Comprende los textos, tipologías, la exposición oral, las estrategias y niveles de comprensión de textos, y la redacción de informes y artículos científicos".

De ella se desprende la competencia de la asignatura "Desarrolla habilidades y estrategias de comunicación oral y escrita en función a sus necesidades y a las características de las actividades académicas que realiza, con sentido crítico y reflexivo". Además se prescriben las competencias de unidad: Unidad I: Ejercita sus habilidades de expresión y comprensión oral en contextos académicos. Unidad II: Utiliza estrategias de expresión y comprensión escrita en función a las tareas y actividades académicas.

El alineamiento constructivo de Habilidades y estrategias de aprendizaje (Métodos y técnicas de estudio), se basó en la psicología cognitiva. Se tienen que desarrollar habilidades y estrategias de aprendizaje en el contexto de la vida universitaria. Más que conocimiento teórico se tienen que ejercitarlas como herramientas cognitivas que permitan generar aprendizajes. Sus contenidos son los procesos mentales señalados por la psicología cognitiva: relación, análisis, síntesis, evaluación, etc., diseñando para ellas actividades con cualquier tema 0 asignatura del currículo: física, matemática, informática o sociales, etc.

Se obtiene como producto el sílabo de la asignatura de Habilidades y estrategias de aprendizaje cuya sumilla está descrita como "Asignatura de naturaleza teórico-práctica y de formación general. Desarrolla en el estudiante las habilidades necesarias para alcanzar un aprendizaje permanente, autónomo y significativo durante su vida universitaria; un aprender a aprender a través del reconocimiento de sus habilidades y la utilización de estrategias de aprendizaje.
Comprende el análisis, la síntesis y la evaluación como procesos cognitivos integradores. La planificación, organización, manejo del tiempo; la búsqueda, procesamiento y análisis de información; la escucha activa, la participación en clase, la toma de apuntes y la preparación para las evaluaciones".

De ella se desprende la competencia de la asignatura "Desarrolla habilidades y estrategias de aprendizaje en función a sus necesidades y a las características de las actividades académicas que realiza en la universidad, regulando sus procesos cognitivos, afectivos y motivacionales". Además se prescriben las competencias de unidad: Unidad I: Ejercita sus procesos cognitivos para consolidarlas como habilidades de aprendizaje. Unidad II: Utiliza estrategias de aprendizaje en función a las tareas y actividades académicas.

\section{DISCUSIÓN}

Sobre los enfoques de aprendizaje que involucran los procesos cognitivos de los estudiantes, se tiene que el $82,98 \%$ presenta un enfoque profundo. Contrariamente, Maquilón (5), encontró que casi la mitad de ingresantes emplean el enfoque superficial, aplicando un cuestionario sobre procesos de estudio a partir del marco teórico de Biggs. En cambio, García (2), halla que un porcentaje pequeño y similar que tienen un enfoque predominantemente profundo 0 predominantemente superficial. La mayoría, está más motivado intrínsecamente, sin embargo utiliza estrategias memorísticas como comprensivas. Para el recojo de datos utilizó el cuestionario sobre enfoques de aprendizaje de Biggs.

El alineamiento constructivo se realizó empleando el método descriptivo observacional, en la línea de un estudio cualitativo. Manrique (3) apela a una investigación descriptiva y cualitativa, eligiendo una carrera como caso único; para comprender el proceso de evaluación del currículo y la 
toma de decisiones para modificar el plan de estudios de una carrera. Utilizó entrevistas y análisis de documentos con matrices de análisis para establecer relaciones e inferencias; instrumentos similares a los de Arévalo (8) y la investigación (plantillas de análisis de contenido).

En esta línea metodológica, Anglas (9) en una investigación exploratoria de tipo cualitativa, utiliza la técnica del análisis de contenido, para determinar qué características del paradigma posmoderno se presentan en el Modelo Curricular del Programa de Bachillerato, procedimiento similar a Manrique (3) y la investigación que se sustenta.

Arévalo (8) caracteriza algunos puntos de un currículo desalineado. Carrascal (1) aplica el modelo instruccional de alineamiento constructivo e interactivo, a los resultados de aprendizaje y desarrollo de competencias básicas a estudiantes de primer semestre. El Modelo representa el proceso enseñanzaaprendizaje desde la mirada del estudiante, el contexto y sus propios objetivos. La diferencia con la presente investigación, radica en el tipo y nivel que se realiza: explicativa cuantitativa y cuasi experimental (con diseño con pre y post test con grupo control)

Goded (4) afirma que un sílabo comunicativo construido en torno a la adquisición de conocimientos gramaticales tiene menos efectividad desde el punto de vista del aumento de la competencia comunicativa que un sílabo elaborado sobre la base de las necesidades comunicativas de los estudiantes, explicitados mediante su elección de temas e intereses de aprendizaje.

Para el caso de Métodos y técnicas de estudio, Maquilón (5), da respuesta a las necesidades de habilidades de aprendizaje de los universitarios, diseñando un programa de intervención, enfatizando en la necesidad que los estudiantes aborden sus aprendizajes desde una perspectiva profunda, lo que hace necesario el desarrollo de un paradigma de aprendizaje reflexivo, crítico e integral.

Finalmente, en ambos sílabos se han incorporado como contenidos algunas competencias genéricas del Proyecto Tuning de América Latina $(10,11)$ : capacidad de análisis y síntesis, capacidad para organizar y planificar el tiempo, capacidad de comunicación oral y escrita, capacidad de aprender, habilidades para buscar, procesar y analizar información procedente de fuentes diversas. Competencias interpersonales: capacidad crítica y autocrítica, capacidad para identificar, capacidad de trabajo en equipo, habilidades interpersonales. Competencias sistémicas: conciernen a los sistemas como totalidad, suponen una combinación de la comprensión, la sensibilidad y el conocimiento, capacidad de motivar y habilidad para trabajar en forma autónoma.

A la luz de los hallazgos, se pueden establecer las siguientes conclusiones: El 82,98\% de los estudiantes de la muestra de estudio, presentan un enfoque profundo de aprendizaje y sólo el 17,02\% presentan un enfoque superficial de aprendizaje. Sin embargo, la intensidad en el enfoque profundo es distinta entre los estudiantes: el $56,91 \%$ muestra una intensidad baja, el 21,28\% presenta una intensidad media y sólo el 4,79\% presenta una intensidad alta. Por otro lado, dentro del enfoque superficial, el $17,02 \%$ de estudiantes muestran una intensidad baja.

Para el alineamiento constructivo de la asignatura de Comunicación se toma como referente la psicología cognitiva. Se fusionan los aportes del enfoque funcional, procesual y, de contenido de las teorías lingüístico comunicativas, enfatizando el aprendizaje de estrategias y habilidades: hablar, escuchar, leer y escribir.

Para el alineamiento constructivo de Métodos y técnicas de estudio, que debe denominarse habilidades y estrategias de aprendizaje, igualmente se toma como referente la psicología cognitiva. Ejercita los procesos: observación, comparación, relación, clasificación, ordenamiento, análisis, síntesis y evaluación en el contexto 


\section{REFERENCIAS BIBLIOGRÁFICAS}

1. Carrascal TS. Integración de tareas SOLO para el desarrollo de competencias básicas en primer semestre de educación superior [Internet]. Granada: Universidad de Granada; 2010. Disponible en: http://tesis.com.es/tribunales/carmonafernandez-marcelo/documentos/ integracion-tareas-solo-desarrollocompetencias-basicas-primer/

2. García BA. Estudio de los enfoques de aprendizaje en estudiantes de Magisterio y Psicopeda-gogía. Electronic journal of research in educational psychology. 2005; 3(6): 109-126.

3. Manrique VL. La evaluación procesual del currículo y su efecto en el plan de estudios de una carrera de pregrado de la Pontificia Universidad Católica del Perú, estudio de caso [Tesis]. Lima: Pontificia Universidad Católica del Perú; 2009.

4. Goded RM. El papel de la gramática en un syllabus comunicativo [Tesis]. Madrid: Universidad Complutense de Madrid; 2002.

5. Maquilón SJ. Diseño y evaluación del diseño de un programa de intervención para la mejora de las habilidades de aprendizaje de los estudiantes universitarios [Tesis]. Murcia: Universidad de Murcia, Facultad de Educación; 2003.

6. Biggs J, Kember D, Leung DYP. The revised two-factor Study Process Questionnaire: R-SPQ-2F. British Journal of Educational Psychology. 2001; (71)1: 133-149.

7. Bahamón IJ. Un modelo para el diseño curricular de las asignaturas de la Universidad: Alineación constructiva entre objetivos, contenidos de aprendizaje, estrategias didácticas y evaluación. Cali: Universidad Instituto Colombiano de Estudios Superiores de Incolda; 2007.

8. Arévalo AR. Teorías de dominio de los docentes sobre el aprendizaje y su expresión en la evaluación de los aprendizajes: un estudio de caso en una Institución Educativa Particular de Lima [Tesis]. Lima: Pontificia Universidad Católica del Perú, Escuela de Posgrado; 2010.

9. Anglas LTM. Presencia del paradigma postmoderno en el modelo curricular del programa del Diploma de Bachillerato Internacional [Tesis]. Lima: Pontificia Universidad Católica del Perú, Escuela de Posgrado; 2009.

10.Tuningal.org [Internet]. Bilbao: Universidad de Deusto; 2012. [actualizado 2012 aug 23; citado 2012 aug 29]. Disponible en: http://www. tuningal.org/es/competencias.

11. González FJ, Wagenaar T. Tuning Educational Structures in Europe. Final Repport. Phase One, Bilbao: Universidad de Deusto; 2003.

Correo electrónico:

fnaupari@continental.edu.pe 